\title{
Myelopathy secondary to ossification of the posterior longitudinal ligament of the thoracic spine treated by anterior decompression and bony fusion
}

\author{
Y Fujimura, Y Nishi, M Nakamura, M Watanabe and M Matsumoto \\ Department of Orthopaedic Surgery, School of Medicine, Keio University, 35 Shinanomachi, Shinjuku-ku, Tokyo, 160 \\ Japan
}

\begin{abstract}
We examined the utility of anterior decompression and bony fusion via the extrapleural approach in the treatment of thoracic myelopathy secondary to ossification of the posterior longitudinal ligament (OPLL). Patient outcome and complications were analyzed in 48 patients treated with this procedure, with a follow-up of at least 2 years. The Japanese Orthopaedic Association score was used to evaluate the severity of the thoracic myelopathy, and the recovery rate was used to evaluate the surgical outcome. The outcome, postoperative complications, radiographic evaluations of bony union, and progression of OPLL within the area of anterior decompression were examined. The T3 vertebral body was the highest level to which anterior decompression was applied. The average follow-up period was 57 months with a recovery rate of $56.7 \%$ which stabilized 1 year after operation. However, the surgical outcome was less favorable in patients with long-standing myelopathy, extensive OPLL, or thoracic OPLL with coexisting intraspinal ligament ossification. Four patients experienced deterioration of their myelopathy, and seven patients had the postoperative complication of extraspinal leakage of cerebrospinal fluid. The myelopathy was transient in all but one patient. Radiographic studies showed that bony union was achieved and restenosis of the spinal canal due to progression of OPLL within the area of decompression did not occur. We conclude that anterior decompression and bony fusion using the extrapleural approach provides a good outcome and is useful in treating mid- and lower thoracic OPLL when performed carefully at an early stage of disease.
\end{abstract}

Keywords: ossification of the posterior longitudinal ligament (OPLL); thoracic myelopathy; thoracic spine; extrapleural approach; anterior decompression and bony fusion

\section{Introduction}

Ossification of the posterior longitudinal ligament (OPLL) of the thoracic spine occurs less frequently than OPLL of the cervical spine, ${ }^{1-3}$ but requires surgical treatment when complicated by myelopathy. In even mild degrees of myelopathy, conservative treatment is ineffective. ${ }^{2,4}$ The surgical procedure employed can be either an anterior or a posterior decompression. A number of studies have examined the indications and results of these two approaches without establishing a definite standard. ${ }^{5-24}$ Since 1979 , we have used anterior decompression and bony fusion with the extrapleural approach to treat mid- and lower thoracic OPLL. We selected this method because the myelopathy associated with thoracic OPLL is caused by an anterior lesion. ${ }^{10,20}$ The purpose of this study was to examine the advantages and disadvantages of

Correspondence: Y Fujimura MD this treatment by analyzing outcomes including complications in patients treated with this procedure.

\section{Materials and methods}

Forty-eight patients with OPLL of the thoracic spine were treated with anterior decompression and bony fusion via the extrapleural approach from 1979 to 1993. They were followed for at least 2 years. There were 18 males and 30 females. The patients ranged in age from 27 to 70 years (mean: 53). The extrapleural approach was used in 46 cases (Figure 1). A two-stage operation was performed of extrapleural and manubrium-splitting approaches in two patients with the upper thoracic OPLL, and was performed of extrapleural approaches in two patients with extensive thoracic OPLL. Expansive open-door laminoplasty was also performed in seven patients who had cervical OPLL, and laminectomy was also performed in six patients with ossification of the ligamentum flavum 
(OLF) (Figure 2). The patients were followed for 25 to 159 months (average: 57 months).

In order to evaluate the severity of thoracic myelopathy, we modified the scoring system for cervical myelopathy established by the Japanese Orthopaedic Association (JOA) by excluding items

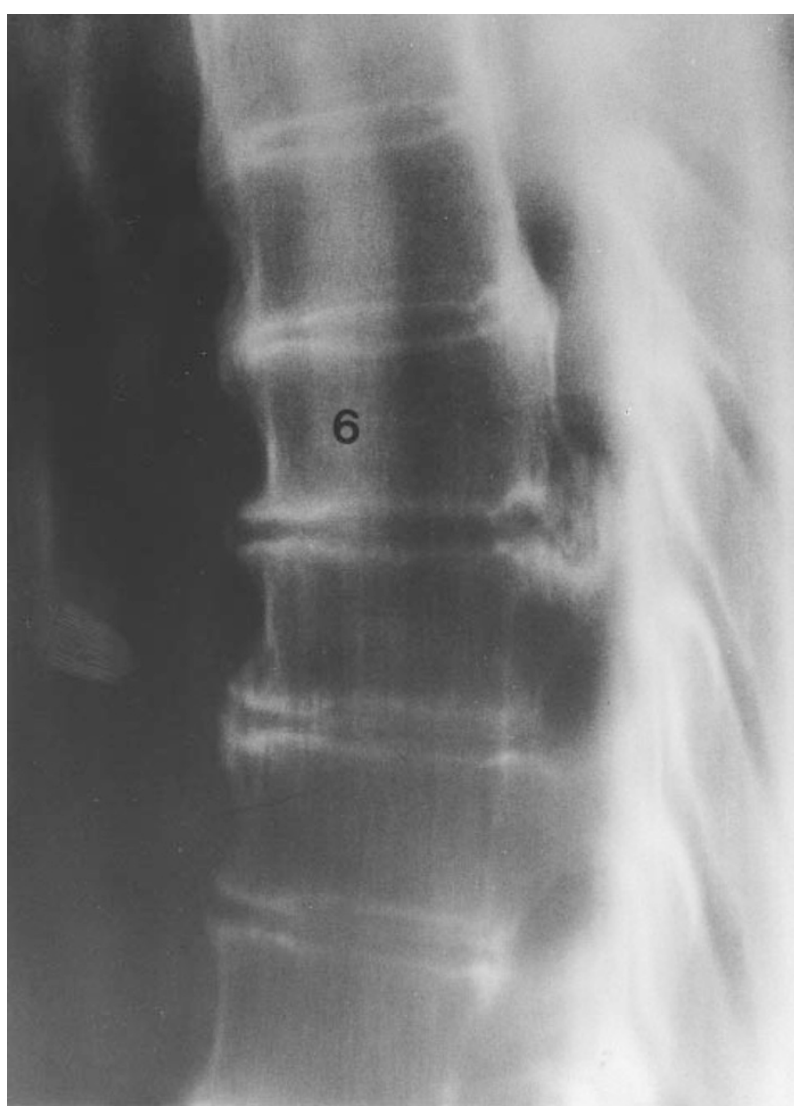

b

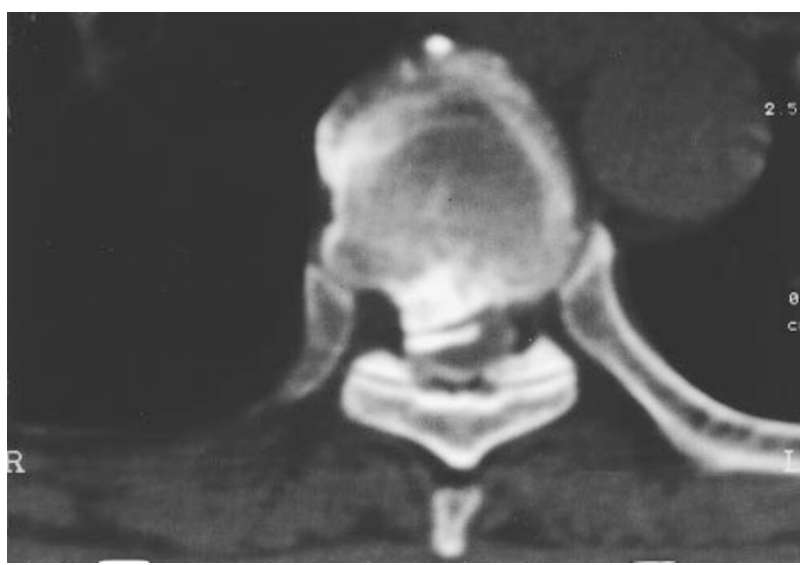

concerning the upper extremities. The modified system has a normal score of 11 points (JOA score) (Table 1). The patient's recovery rates were calculated from the preoperative and postoperative JOA scores, while the postoperative JOA score alone was used to evaluate surgical outcome. The JOA score was

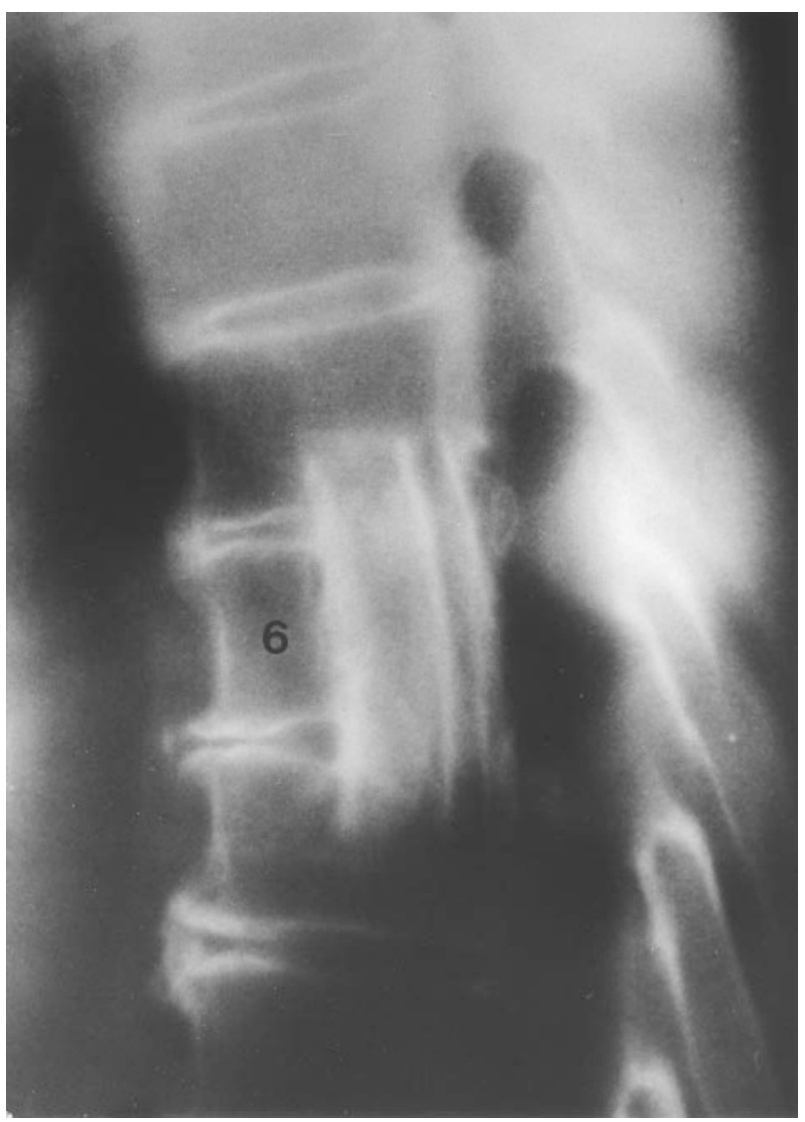

d

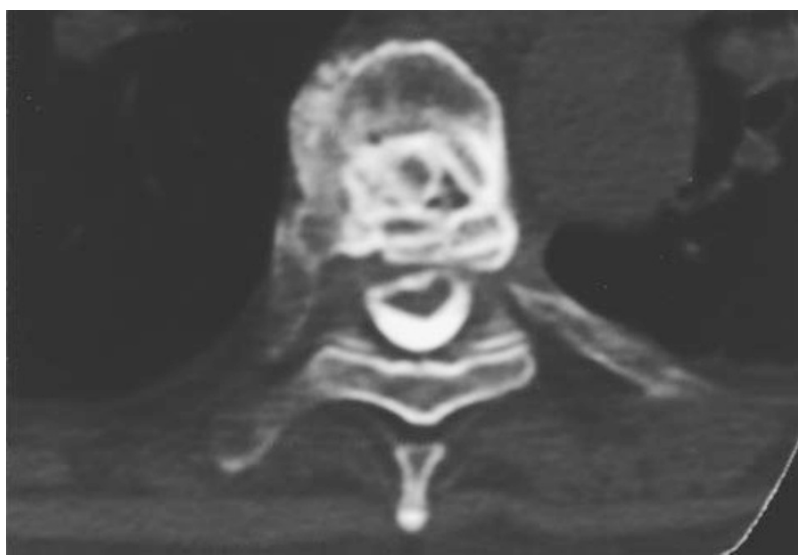

Figure 1 The radiographic findings of a 62-year-old female with OPLL of T4-7 for whom an anterior decompression and bony fusion of T5-7 was performed using the extrapleural approach. (a) Preoperative lateral tomogram showing OPLL of T4-7. (b) Preoperative computed tomographic myelogram (CTM) showing OPLL of T6. (c) Postoperative lateral tomogram showing anterior decompression and bony fusion of T5-7. (d) Postoperative CTM showing anterior decompression and bony fusion of T6 
measured and the recovery rate determined at 3 months and again at 1 year after operation as well as at the final follow-up examination. The final outcomes were classified as excellent, good, fair, unchanged, or worse based on recovery rates of $75 \sim 100 \%, \quad 50 \sim 74 \%, \quad 25 \sim 49 \%, \quad 0 \sim 24 \%$, or less than $0 \%$, respectively. The relationships between the recovery rate and the following factors were investigated statistically using a nonparametric test followed by a Mann - Whitney $U$ test: age at surgery, the severity of myelopathy, the duration of myelopathy, the degree of spinal canal stenosis, the number

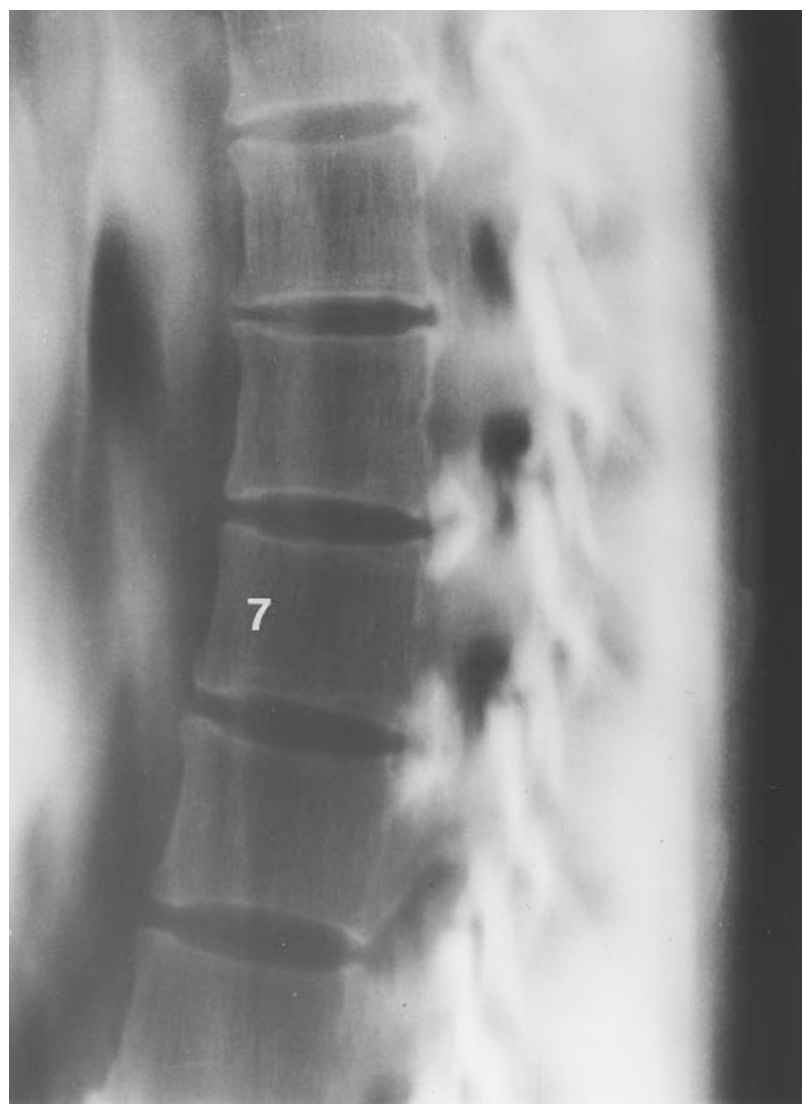

b

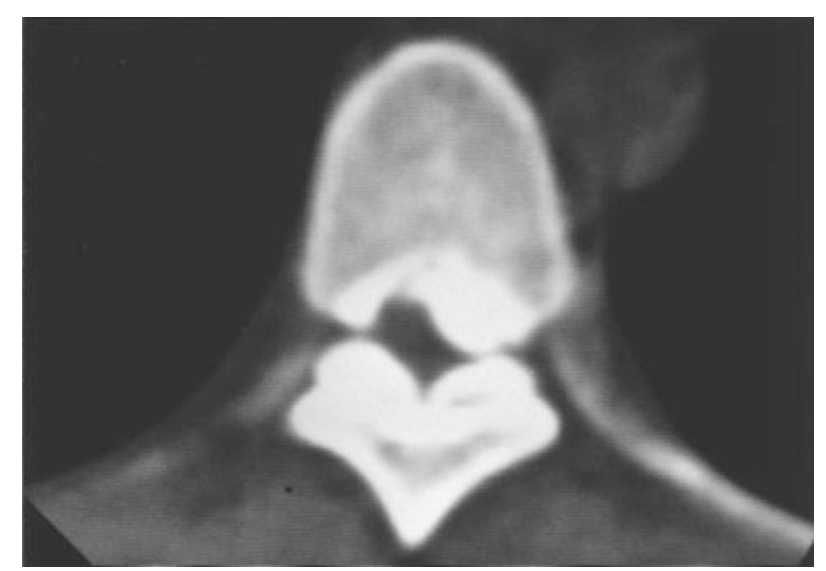

C

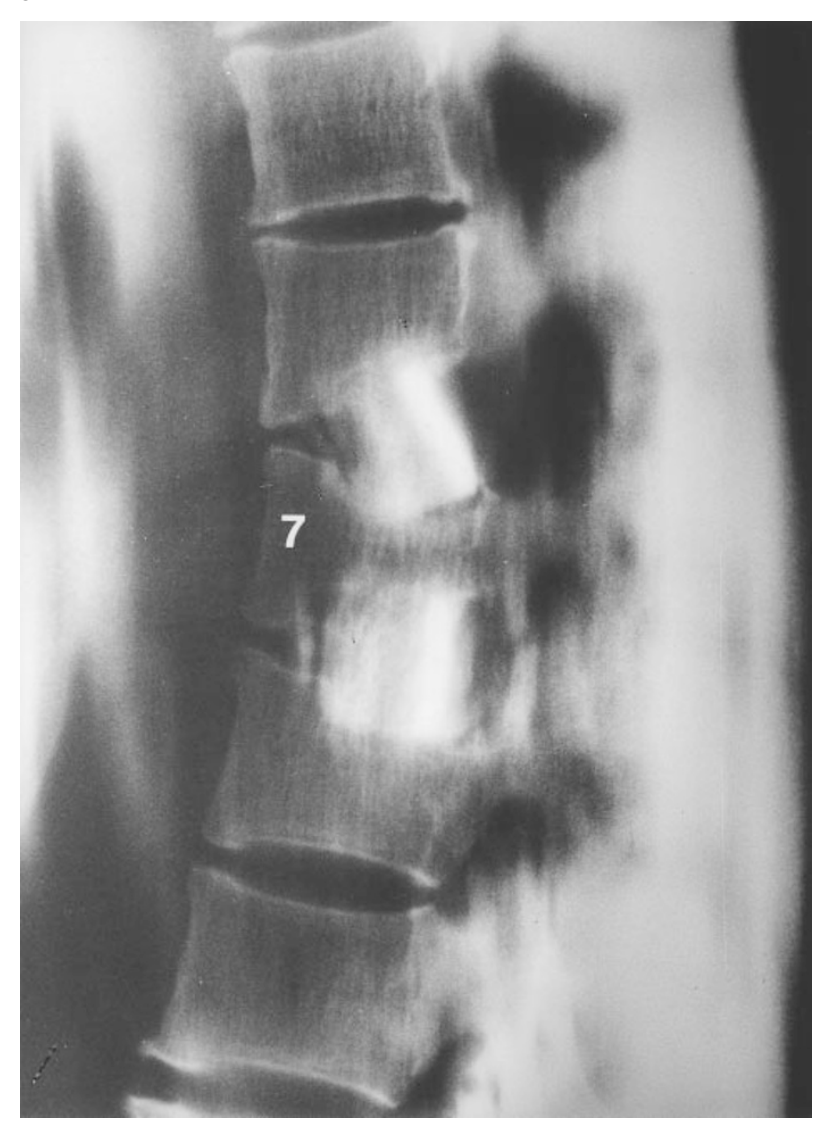

d

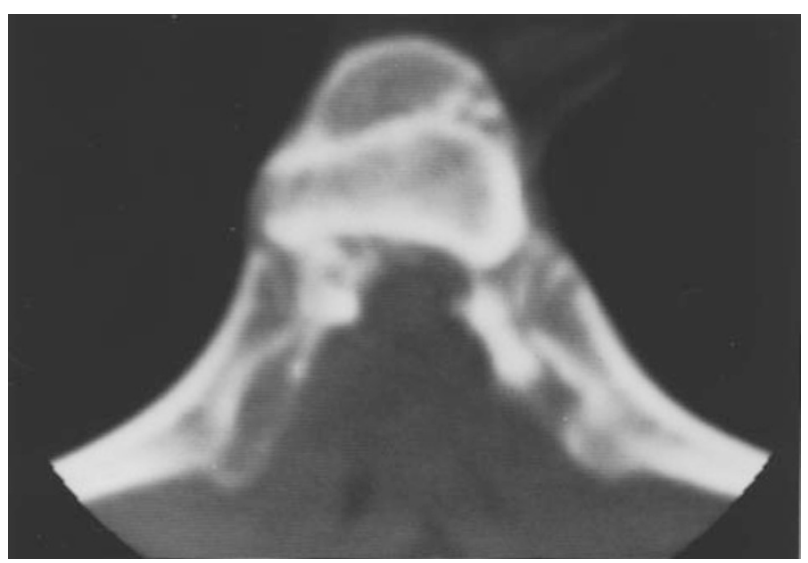

Figure 2 The radiographic findings of a 36-year-old female with OPLL of T5-8 and OLF of T5-9. An anterior decompression and bony fusion of T6-T8 and a posterior decompressive laminectomy of T5-8 were performed. (a) Preoperative lateral tomogram showing OPLL of T5-8 and OLF of T5-9. (b) Preoperative computed tomogram (CT) showing both OPLL and OLF of T7. (c) Postoperative lateral tomogram showing anterior decompression and bony fusion of T6-8 with posterior decompressive laminectomy of T5-8. (d) Postoperative CT showing anterior decompression and bony fusion with posterior decompressive laminectomy of T7 
Table 1 Evaluation system for thoracic myelopathy (Japanese Orthopaedic Association score)

\begin{tabular}{lc}
\hline Category & Score (point) \\
\hline A. Lower extremity motor function & 0 \\
Unable to stand up and walk by any means & 1 \\
Unable to walk without a cane or other support on a level & 2 \\
Walks independently on a level but needs support on stairs & 3 \\
Capable of fast walking but clumsily & 4 \\
Normal & 0 \\
B. Sensory function & 1 \\
I. Lower extremity & 2 \\
Apparent sensory loss & \\
Minimal sensory loss & 0 \\
Normal & 1 \\
II. Trunk & 2 \\
Apparent sensory loss & \\
Minimal sensory loss & \\
Normal & 1 \\
Urinary retention and/or incontinence & 2 \\
Sense of retention and/or dribbling and/or thin stream & 3 \\
Urinary retardation and/or pollakiuria & \\
Normal & \\
\hline
\end{tabular}

Total for normal patient 11

Recovery rate $\% \quad \frac{\text { postoperative score preoperative score }}{11 \text { preoperative score }} 100$

of vertebral bodies decompressed and the presence of coexisting intraspinal ligament ossification (cervical OPLL or OLF). The degree of preoperative severity was determined by lower extremity motor dysfunction, and the presence of intraspinal ligament ossification seen at operation. In addition, we monitored the incidence of postoperative complications and used radiographic studies to follow bony union and OPLL progression within the area of anterior decompression.

\section{Indications}

The indications for surgery were based on the severity of the thoracic myelopathy, with particular emphasis on the degree of lower extremity motor dysfunction and bladder dysfunction. A JOA score of two or less for lower extremity motor function, or two or less for bladder function, were considered absolute indications for surgery. The preoperative JOA scores ranged from 0 to 6 points (average: 3.4 ) in the 48 patients.

\section{Surgical approach and technique of anterior}

decompression and bony fusion

The patient was placed in the lateral position with the arm on the involved side raised. The operating table was flexed at the level of T3 to T12 to afford good exposure and to facilitate the procedure. A left-sided anterior approach was usually used, although either side may be used. A skin incision was made along the line of the rib that corresponded to the thoracic vertebra one or two levels above the involved

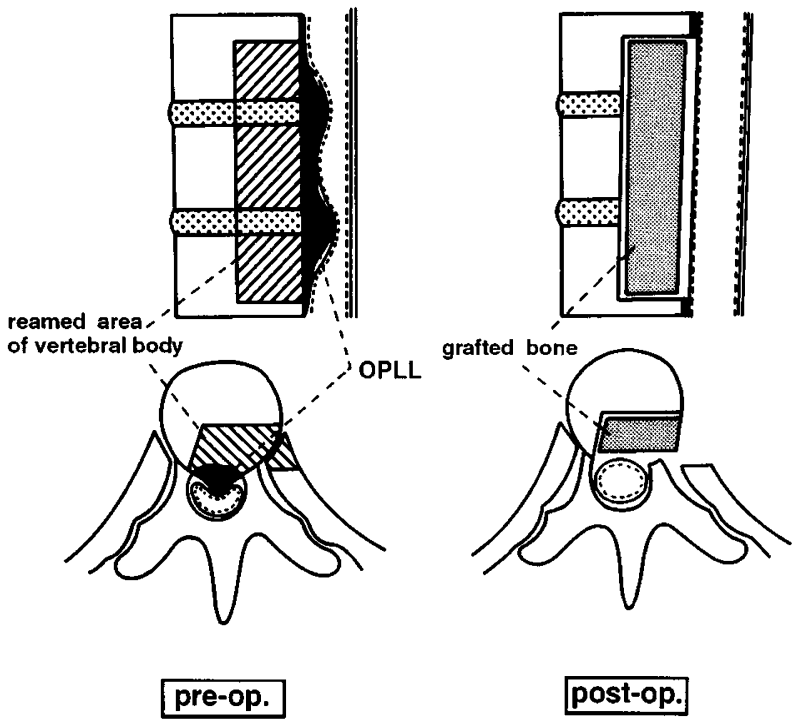

Figure 3 Schematic illustration of anterior decompression and bony fusion for OPLL of the mid- and lower thoracic spine via the extrapleural approach

vertebra. The rib was exposed and excised, and the pleura was stripped from the chest wall as extensively as possible. The lung and great vessels were retracted to expose the anterior and lateral side of the thoracic vertebral body so that the head of the rib could be excised. A steel burr was used to resect the posterior half of the vertebral body (three quarters in width). 
After the OPLL was exposed, it was planed thin and removed. When the OPLL was adherent to the dura mater, the anterior floating method was used. ${ }^{25}$ Once the anterior decompression was completed, anterior bony fusion was carried out with an iliac crest or rib graft (Figure 3).

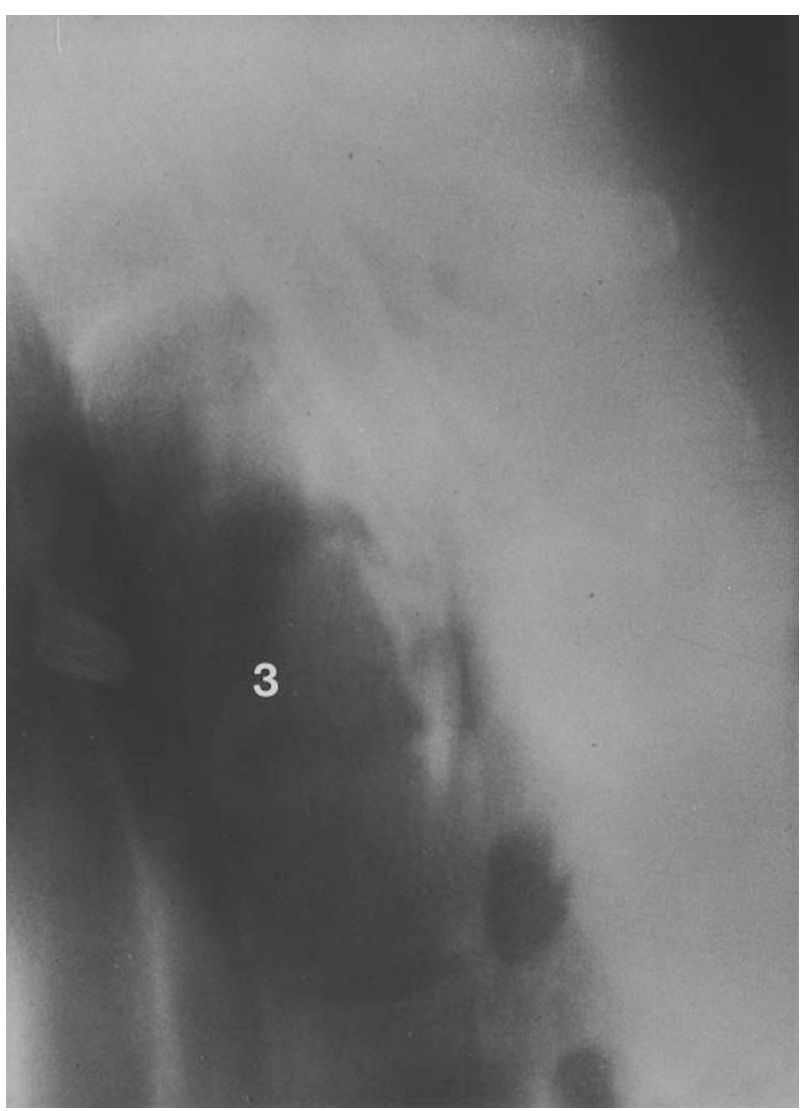

b

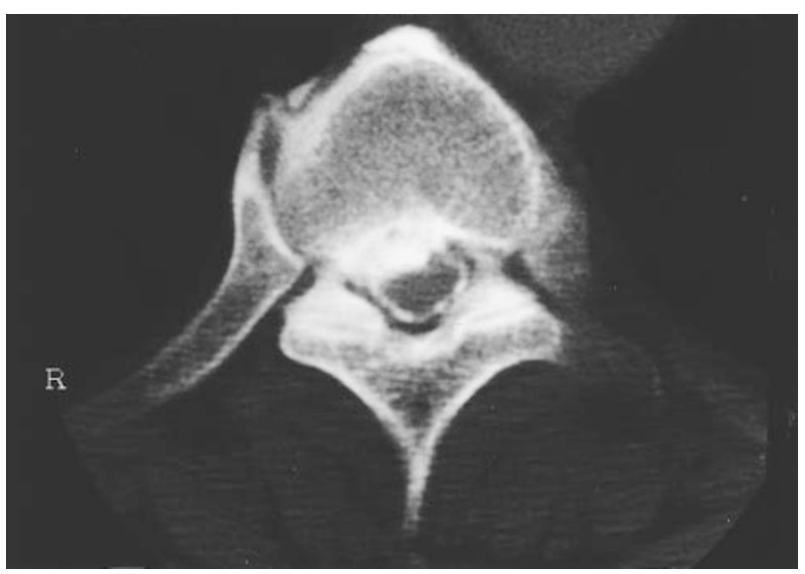

\section{Postoperative care}

The patients were confined to bed in the supine position. Three weeks after the operation they were equipped with a plaster spinal orthosis and were permitted to leave their beds. The patients were kept in the orthosis for 12 to 16 weeks.

C

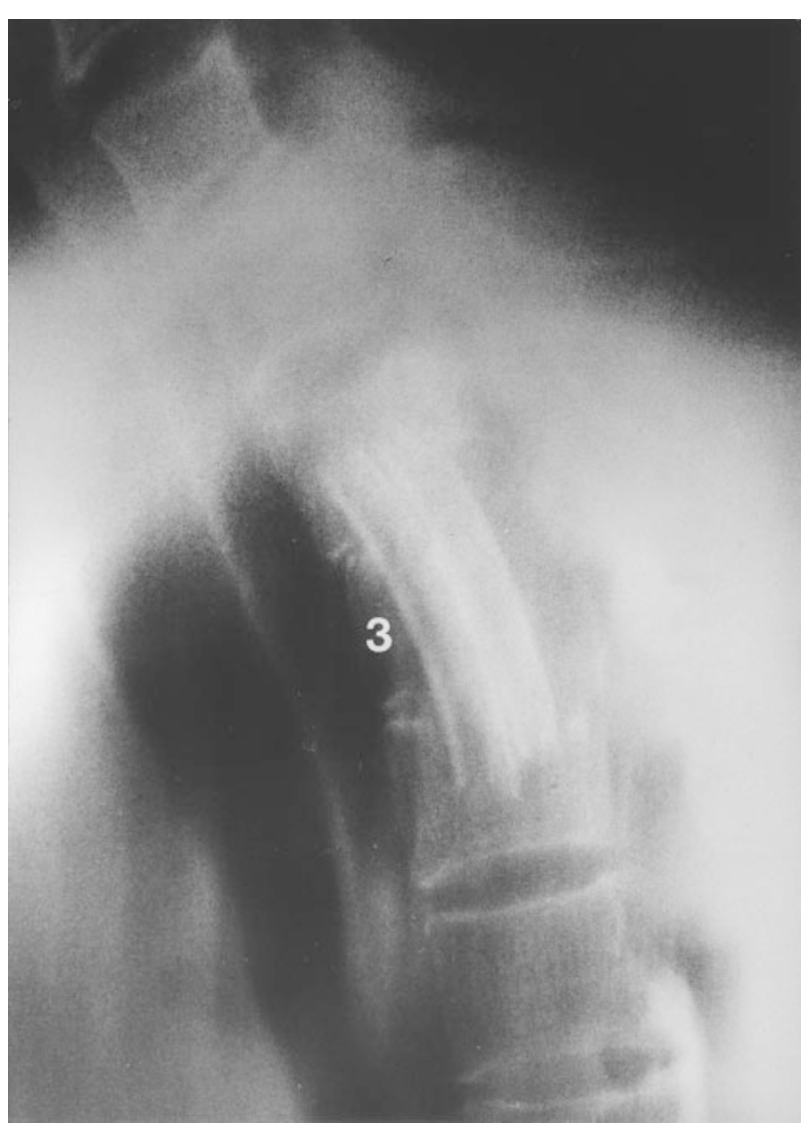

d

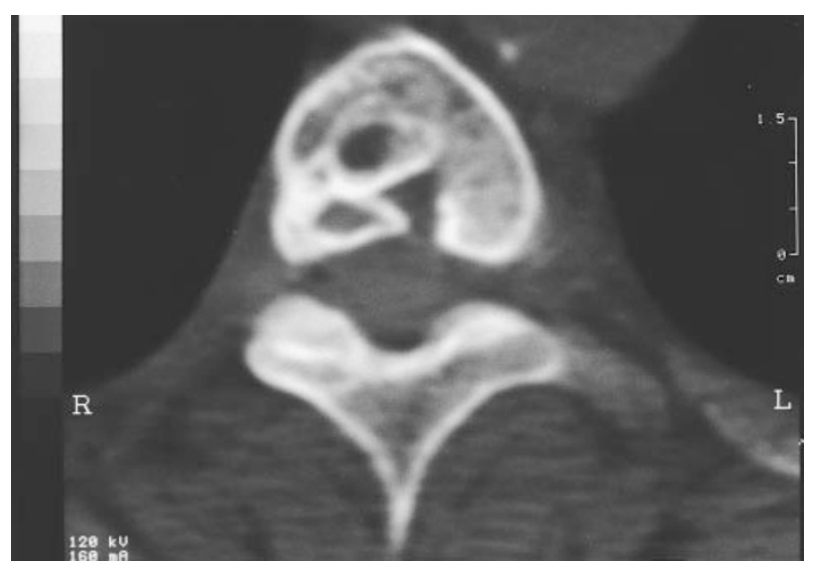

Figure 4 The radiographic findings of a 43-year-old female with OPLL of T2-4 and T5-6. Anterior decompression and bony fusion of T2-4 was performed using the extrapleural approach. (a) Preoperative lateral myelogram showing an incomplete block at T2-3. (b) Preoperative CTM showing OPLL of T3. (c) Postoperative lateral tomogram showing anterior decompression and bony fusion of T2-4. (d) Postoperative CT showing anterior decompression and bony fusion of T3 


\section{Results}

The extent of the anterior decompression was two vertebral bodies (one intervertebral space) in 14 cases, three vertebral bodies in 23 cases, four vertebral bodies in seven cases, five vertebral bodies in three cases and six vertebral bodies in one case. The highest vertebra decompressed with the extrapleural approach was at the T3 level (Figure 4). Because of the upper thoracic OPLL which expanded to both the cephalad and caudad sides of the T3 vertebral body, a two-stage operation of the extrapleural and manubrium-splitting approaches was performed in two patients who underwent a five level decompression. Because of the extensive thoracic OPLL, a two-stage operation of the extrapleural approaches was performed in two patients who underwent five or six level decompression. The average time required to perform a three level decompression was in the range of $3 \mathrm{~h} 34 \mathrm{~min}$ to $5 \mathrm{~h}$ $48 \mathrm{~min}$ (average: $4 \mathrm{~h} 24 \mathrm{~min}$ ). The amount of intraoperative blood loss ranged from 625 to $2729 \mathrm{~g}$ (average: $1320 \mathrm{~g}$ ).

The JOA score preoperatively, at 3 months, at 1 year after the operation, and at the final follow-up was $3.5 \pm 1.9$ (mean \pm SD) points, $6.8 \pm 1.9$ points, $7.9 \pm 1.9$ points, and $7.8 \pm 2.0$ points, respectively. The recovery rate was $44.2 \pm 20.8 \%, 58.4 \pm 22.3 \%$ and $56.7 \pm 23.4 \%$ at 3 months, 1 year after the operation, and at final follow-up, respectively. The average recovery rate was maintained 1 year after surgery (Table 2). The final outcome was excellent in seven patients, good in 24 patients, fair in 11 patients, unchanged in five patients and worse in one patient.

The relationships between the recovery rate and various factors were examined based on the final follow-up. The recovery rate was $57.8 \pm 26.5 \%$ for patients below the age of 60 years $(n=36)$ and $53.8 \pm 13.4 \%$ for those at or above 60 years $(n=12)$, not a significant difference. The recovery rate was $58.4 \pm 23.4 \%$ for patients with a lower extremity motor function of one to two points $(n=37)$ and $54.5 \pm 22.3 \%$ for those with a lower extremity motor function of 0 points $(n=11)$, not a significant difference. The recovery rate was $65.0 \pm 15.8 \%$ for patients suffering for less than 1 year $(n=33)$ and $38.8 \pm 25.3 \%$ for those suffering for 1 year or more $(n=15), \quad$ a significant difference $(P<0.01)$. The recovery rate was $59.6 \pm 26.8 \%$ for patients with a less than $50 \%$ maximum stenosis $(n=27)$ and
$53.2 \pm 17.2 \%$ for those with a $50 \%$ or greater maximum stenosis $(n=21)$, not a significant difference. The recovery rate was $67.8 \pm 17.1 \%$ for two vertebral body decompressions $(n=14)$ and $52.2 \pm 21.6 \%$ for three or more vertebral body decompressions $(n=34)$, a significant difference $(P<0.05)$. The recovery rate was $61.5 \pm 17.5 \%$ for those patients who underwent an anterior thoracic decompression $(n=35)$ and $44.2 \pm 19.8 \%$ for those who underwent a combined operation for cervical OPLL or OLF $(n=13)$, a significant difference $(P<0.05)$ (Figure 5).

The complications included four patients with deterioration in their thoracic myelopathy and seven who had extrapleural leakage of cerebrospinal fluid. Three of the patients who deteriorated neurologically improved after 2 weeks, and six of the patients with a cerebrospinal fluid leak were healed by 6 weeks. The extrapleural cerebrospinal fluid leak in the remaining patient was treated with a dura mater repair. Radiographic studies indicated bony union in all patients, and spinal canal restenosis from progression of OPLL within the area of anterior decompression was not observed.

\section{Discussion}

The effects of dynamic forces on the thoracic cord are small, as the thoracic spine is anatomically stabilized by the presence of the rib cage. The thoracic spinal canal is narrow and blood flow to the thoracic cord is minimal. ${ }^{26}$ The concurrent presence of functional stability of the spine and the physiological vulnerability of the thoracic cord suggest that the progression and severity of a myelopathy would likely be rapid and severe once it occurs. In addition, as the role of static factors, such as spinal cord compression, is greater than the role of dynamic factors, the reliability of conservative therapy is minimal, and early surgical intervention is often indicated.

The objective of surgical treatment is the complete decompression of the spinal cord. This can be achieved by removing the OPLL in an anterior decompression. This is the logical treatment of thoracic OPLL, although a posterior decompression is frequently selected because the surgical manoeuvers required for anterior decompression are difficult. ${ }^{5-7,9,11,16,21}$ However, the physiological kyphosis of the thoracic spine renders the decompressive effect of posterior proce-

Table 2 Preoperative and postoperative JOA scores and recovery rates

\begin{tabular}{|c|c|c|c|c|}
\hline & Preoperative & 3 months & $\begin{array}{c}\text { Postoperative } \\
1 \text { year }\end{array}$ & Final follow-up \\
\hline $\begin{array}{l}\text { JOA score (points) } \\
\text { Recovery rate }(\%)\end{array}$ & $3.5 \pm 1.9$ & $\begin{array}{c}6.8 \pm 1.9 \\
44.2 \pm 20.8\end{array}$ & $\begin{array}{c}7.9 \pm 1.9 \\
58.4 \pm 22.3\end{array}$ & $\begin{array}{c}7.8 \pm 2.0 \\
56.7 \pm 23.4\end{array}$ \\
\hline
\end{tabular}

Postoperative JOA scores at each follow-up increased significantly compared to preoperative scores $(P<0.01)$. Significantly better JOA scores and recovery rates were obtained at 1 year or more than those at 3 months after the operation $(P<0.01)$. Final follow-up was performed an average of 4 years and 9 months after the operation 


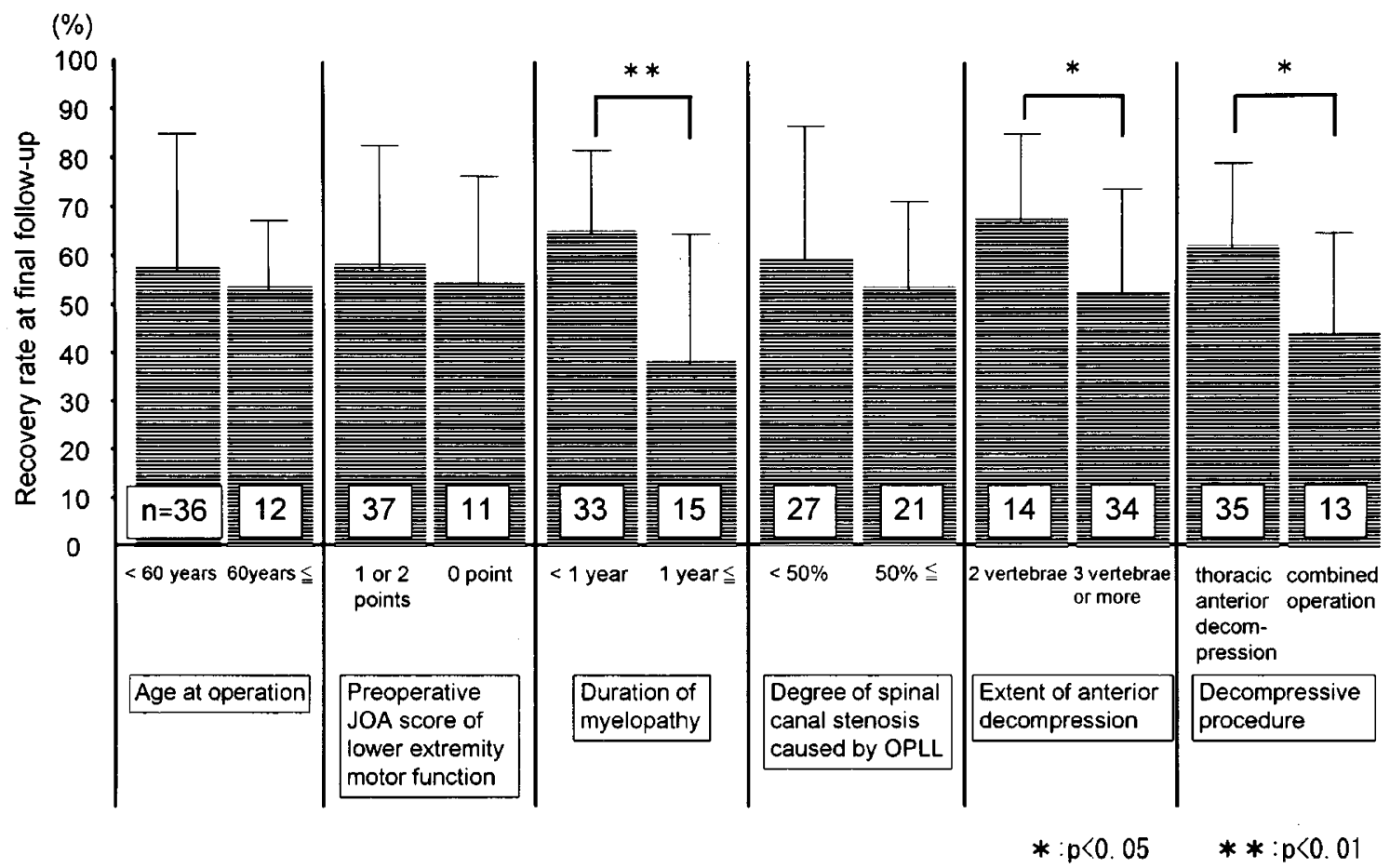

Figure 5 The relationship between recovery rates and various patient factors. Age at operation, preoperative severity of myelopathy, or degree of spinal canal stenosis did not significantly affect recovery rate. However, a long duration of myelopathy was associated with a lower recovery rate $(P<0.01)$ as was the need for a combined operation $(P<0.05)$. A better recovery rate was seen when the extent of the OPLL was less than three vertebrae $(P<0.05)$

dures unreliable. Kyphosis also may increase after a posterior decompression and cause a further deterioration of myelopathy. Therefore, posterior decompression should be avoided. ${ }^{8,10,12,17,20,22,23}$

The approach used for an anterior decompression can be from an anterior, ${ }^{8,10,12,17,20}$ posterior, ${ }^{14,18,19}$ or lateral ${ }^{8,23}$ direction. The indications and outcomes of these approaches have been studied and reported, but no approach is well established at present. We examined the postoperative outcome of an anterior decompression and bony fusion performed via the extrapleural approach, in order to clarify both the advantages and the problems of this procedure. Analysis of our 48 patients showed favorable clinical and radiographic outcomes. In addition, there were no severe complications. However, it should be noted that anterior decompression by our method could not extend beyond T3 vertebral body. Although postoperative outcomes suggest that our method is strongly indicated for localized OPLL, the range of decompression was limited by anatomic restrictions. In fact, a two-stage operation was required when decompression at five or more vertebral levels was anticipated because of the upper thoracic OPLL expanding above T3 level, and because of the extensive thoracic OPLL.

The factors affecting surgical outcome included the duration of the myelopathy, the extent of the anterior decompression, and whether a combined operation for cervical OPLL and OLF was required. Chronic myelopathy, extensive OPLL, and coexisting intraspinal ligament ossification predict a negative outcome. We believe that the outcome for localized OPLL was good because the level of ossification was easily determined, and performing the operation at an early stage and facilitated adequate decompression. In contrast, the outcomes for extensive OPLL and, for thoracic OPLL combined with cervical OPLL or OLF, were poorer because it was difficult to determine the level of decompression, and the decompression was inadequate.

A number of patients experienced neurological complications or cerebrospinal fluid leakage, indicating that anterior decompression must be performed with particular attention to preventing complications. If the OPLL and the dura mater are found to be adherent, forceful OPLL resection should be avoided, and the anterior floating method should be substituted.

\section{Conclusion}

We studied the outcomes and complications of 48 thoracic OPLL patients treated with anterior decompression and bony fusion via the extrapleural approach. This procedure resulted in positive outcomes, indicating that it is useful when performed in a 
careful manner at an early stage of thoracic myelopathy. However, limitations to this procedure were also found in treating extensive OPLL, and OPLL with coexisting intraspinal ligament ossification. Furthermore, the cranial limit for anterior decompression was at the level of the T3 vertebral body.

\section{References}

1 Terayama K. Ossification of the posterior longitudinal ligament of the spine. J Jpn Orthop Assoc 1976; 50: 415-442.

2 Tsuyama N, Kurokawa T. Statistical analysis of the patients with ossification of the posterior longitudinal ligament of the thoracic and lumbar spines. Rinsho Seikei Geka 1977; 12: 337 - 339.

3 Tsuyama N. Ossification of the posterior longitudinal ligament of the spine. Clin Orthop 1984; 184: $71-84$.

4 Kurokawa T et al. Diagnosis of the thoracic spinal canal stenosis and the evoked spinal cord action potential measurement. Rinsho Seikei Geka 1981; 16: $32-42$.

5 Forcier P, Horsey WJ. Calcification of the posterior longitudinal ligament at the thoracolumbar junction: case report. $J$ Neurosurg 1970; 32: $684-685$.

6 Imai T et al. Clinical evaluation of ossification of the posterior longitudinal ligament of the thoracic spine. Rinsho Seikei Geka 1977; 12: $340-344$

7 Murakami Y et al. Posterior decompression for thoracic myelopathy due to ossification of the posterior longitudinal ligament. Rinsho Seikei Geka 1977; 12: 345 - 352.

8 Ohtani K, Manzoku S, Shibasaki K, Nomachi S. Anterior decompression for ossification of the posterior longitudinal ligament of the thoracic spine. Rinsho Seikei Geka 1977; 12: $353-359$.

9 Miyazaki K et al. Surgical treatment for ossification of the posterior longitudinal ligament of the thoracic spine. Rinsho Seikei Geka 1977; 12: 360 - 367.

10 Fujimura Y et al. Surgical treatment for thoracic myelopathy due to spinal canal stenosis. Rinsho Seikei Geka 1981; 16: 51-62.

11 Kaneda K et al. Thoracic spinal canal stenosis due to ossification of the spinal canal ligament. Rinsho Seikei Geka 1981; 16: 63-74.

12 Ohtani K et al. Anterior surgical decompression for thoracic myelopathy as a result of ossification of the posterior longitudinal ligament. Clin Orthop 1982; 166: $82-88$.
13 Hanai K et al. Decompression of the OPLL in the thoracic spine using the lateral approach. Seikeigeka 1982; 33: 1059-1062.

14 Ohtsuka $\mathrm{K}$ et al. A surgical procedure for the anterior decompression of the thoracic spinal cord through the posterior approach. Orthop Surg Traumatol 1983; 26: $1083-1090$.

15 Sato T, Kokubun S. Surgical treatment for thoracic myelopathy due to ossification of the posterior longitudinal ligament and ligamentum flavum. Orthop Surg Traumatol 1983; 26: $1993-$ 2001.

16 Sakou T et al. Results of surgical treatment for the ossification of yellow ligament and posterior longitudinal ligament of the thoracic and thoracolumbar spine. Seikeigeka 1985; 36: $1367-$ 1375.

17 Yonenobu $\mathrm{K}$ et al. Thoracic myelopathy secondary to ossification of the spinal ligament. J Neurosurg 1987; 66: 511-518.

18 Tsuzuki N, Tanaka H, Seichi A. Laminopediculoplasty: a new method of reconstructing the posterior elements of the thoracic spine. International Orthopaedics 1989; 13: 39-45.

19 Kataoka O, Sho T, Nishibayashi Y. A circumferential decompression of spinal cord through posterior approach for myelopathy due to the ossification of posterior longitudinal ligament on the upper thoracic level. Seikeigeka 1989; 40: 669675.

20 Fujimura Y, Satomi K, Hirabayashi K. Anterior decompression for thoracic myelopathy due to ossification of the posterior longitudinal ligament. Spine 1989; 2: 671-677.

21 Abumi K, Kaneda K, Hatayama A. Indication and limitation of the posterior decompressive procedures for ossification of the posterior longitudinal ligament of the thoracic region. Spine 1989; 2: $678-682$.

22 Tomita $\mathrm{K}$ et al. Circumspinal decompression for thoracic myelopathy due to combined ossification of the posterior longitudinal ligament and ligamentum flavum. Spine 1990; 15: $1114-1120$

23 Yonenobu $\mathrm{K}$ et al. Lateral rhachotomy for thoracic spinal lesions. Spine 1990; 15: $1121-1125$.

24 Tsuzuki $\mathrm{N}$ et al. Extensive cervicothoracic laminoplastic decompression for the thoracic myelopathy due to OPLL. Rinsho Seikei Geka 1993; 28: $303-311$.

25 Yamaura I. Anterior decompression for the OPLL myelopathy. Anterior floating method of OPLL. Rinso Seikei Geka 1983; 18: $855-868$

26 Dommisee GF. The blood supply of the spinal cord: a critical vascular zone in spinal surgery. J Bone Joint Surg 1974; 56B: $225-235$ 